\title{
Updating the Canadian clinical practice guideline for managing pediatric obesity: a protocol
}

\author{
Bradley C. Johnston PhD, Roah Merdad MBBS MSc, Diana Sherifali PhD RN, Maryam Kebbe PhD, \\ Catherine S. Birken MD MSc, Annick Buchholz PhD, Long Ge PhD, Nicole D. Gehring MSc, \\ Stasia Hadjiyannakis MD, Jill Hamilton MD, Dawn Hatanaka MA, Mélanie Henderson PhD MD, \\ Tracy Lebel, Sarah A. Moore PhD, Katherine M. Morrison MD, Ximena Ramos Salas PhD, \\ Meghan Sebastianski PhD, Ian S. Zenlea MD MPH, Geoff D.C. Ball PhD RD
}

Abstract

Background: Since the first national guideline for managing obesity in adults and children in Canada was published in 2007, new evidence has emerged and guideline standards have evolved. Our purpose is to describe the protocol used to update the Canadian clinical practice guideline for managing pediatric obesity.

Methods: This guideline will update the pediatric components of the 2007 Canadian clinical practice guideline for the management of obesity. In partnership with Obesity Canada, we began preliminary work in 2019; activities are scheduled for completion in 2022. The guideline will follow standards developed by the National Academy of Medicine and the Grading of Recommendations, Assessment, Development and Evaluation (GRADE) working group. Guideline development will be informed by 5 complementary literature reviews: a scoping review that focuses on clinical assessment in pediatric obesity management and 4 systematic reviews to synthesize evidence regarding families' values and preferences as well as the safety and effectiveness of interventions (psychological and behavioural; pharmacotherapeutic; and surgical). We will use standard systematic review methodology, including summarizing and assessing the certainty of evidence and determining the strength of recommendations. Competing interests will be managed proactively according to recommendations from the Guidelines International Network. Diverse stakeholders, including families and clinicians, will be engaged throughout guideline development.

Interpretation: The guideline will support Canadian families and clinicians to make informed, value-sensitive and evidence-based clinical decisions related to managing pediatric obesity. The guideline and accompanying resources for end-users will be published in English and French, and we will partner with Obesity Canada to optimize dissemination using integrated and end-of-project knowledge translation.

$\mathrm{P}$ ediatric obesity is a global health issue. Worldwide, the number of 5- to 19-year-olds with obesity increased from 11 million in 1975 to 124 million in $2016 .{ }^{1}$ In Canada, levels remain high, with approximately one-quarter of 4- to 11-year-olds and one-third of 12- to 17-year-olds classified as having overweight or obesity..$^{2-4}$ This high prevalence is concerning as metabolic, mechanical and mental health issues as well as social milieu concerns are common in children and adolescents with obesity. ${ }^{5}$ Further, obesity seldom resolves spontaneously, ${ }^{6,7}$ and longer exposure to obesity is associated with prolonged and increased morbidity. ${ }^{8}$ As such, there is an imperative to develop and evaluate interventions that are effective for managing pediatric obesity.

Since the first Canadian clinical practice guideline on managing and preventing obesity in adults and children was published in 2007, ${ }^{9}$ several pediatric-specific guidelines have been published, in Canada ${ }^{10}$ and internationally. ${ }^{11,12}$ These guidelines were based on evidence regarding the potential benefits and harms of obesity management strategies; however, there is a need to update existing recommendations because new evidence has accumulated and methodologic approaches have evolved over recent years. As highlighted in the 2020 Canadian guideline for adult obesity, ${ }^{13}$ managing obesity extends beyond addressing weight; management includes a host of outcomes (e.g., mental health) and issues (e.g., obesity stigma) that reflect a broad view of health and social well-being. Historically, guidelines have emphasized treatment effects and seldom included families and clinicians

Competing interests: See the end of the article.

This article has been peer reviewed.

Correspondence to: Geoff Ball, gdball@ualberta.ca

CMAJ Open 2022 March 1. DOI:10.9778/cmajo.20200289 
as stakeholders in developing, refining and evaluating recommendations on the basis of stakeholder values and preferences.

The purpose of this protocol is to describe the organizational approach and methodologic strategies used to update the 2007 Canadian clinical practice guideline for managing pediatric obesity. With a focus on obesity management exclusively, we will direct our resources to provide optimal value and preference-sensitive, evidence-based guidance for families and clinicians to manage pediatric obesity, according to international standards for guideline production.

\section{Methods}

We will follow guideline standards set by the National Academy of Medicine (formerly the Institute of Medicine), ${ }^{14}$ the Grading of Recommendations Assessment, Development and Evaluation (GRADE) working group ${ }^{15-17}$ and the Guidelines International Network. ${ }^{18}$ These methods promote transparency, clear and ongoing management of competing interests, engagement of diverse stakeholders, use of systematic review methodology to synthesize all existing evidence addressing the area(s) of inquiry and use of explicit methods for determining the strength of recommendations (Box 1). Resources from the Alberta Health Services Chair in Obesity Research and Obesity Canada will be used to develop the guideline and support knowledge translation and dissemination activities.

\section{Composition of participating groups}

The guideline steering committee ("the committee") will be responsible for guiding all activities, overseeing finances, granting final acceptance of the guideline recommendations and disseminating the finalized recommendations. The committee will also oversee the development of the research questions, the literature reviews and the development of the guideline methods and will vote on the final recommendations, as well as manage any competing interests among members.

\section{Box 1: Certainty of evidence ${ }^{19}$}

GRADE* $^{*}$ and definition

- High: We are very confident that the true effect lies close to that of the estimate of the effect.

- Moderate: We are moderately confident in the effect estimate. The true effect is likely to be close to the estimate of the effect, but there is a possibility that it is substantially different.

- Low: Our confidence in the effect estimate is limited. The true effect may be substantially different from the estimate of the effect.

- Very low: We have very little confidence in the effect estimate. The true effect is likely to be substantially different from the estimate of effect.

Note: GRADE = Grading of Recommendations Assessment, Development and Evaluation.

*Although certainty of evidence is a continuum, GRADE uses discrete categorization, which introduces a degree of subjectivity. Nevertheless, the advantages of simplicity and transparency outweigh these limitations.
In early 2019, the committee chair (G.D.C.B.) and Obesity Canada invited people with clinical and research expertise in pediatric obesity, systematic reviews and guideline development to join the committee. At this time, we also contacted about 30 provincial and national stakeholder groups to raise awareness of our plan to update the guideline and identify opportunities for collaboration and partnership.

Over time, additional committee members, including clinicians and caregivers of children and adolescents with obesity, were recruited from within and beyond multidisciplinary pediatric obesity management clinics; these include 1 professional-public association representative, 2 clinicians (1 general pediatrician and 1 general pediatrician who is an adolescent medicine specialist), 3 caregivers, $5 \mathrm{PhD}$ researchers and 9 clinician-researchers (6 pediatric endocrinologists, 1 psychologist, 1 registered dietitian and 1 registered nurse).

Each month, the committee meets formally by videoconference with ad hoc correspondence as needed. The committee chair (G.D.C.B.) is responsible for assembling the committee, coordinating meetings, supporting authors who will be leading or coleading the systematic reviews with researchers in the McMaster Evidence Review and Synthesis Team (MERST; McMaster University, Hamilton, Ontario) and the Alberta Strategy for Patient-Oriented Research SUPPORT Unit (AbSPORU; University of Alberta, Edmonton) and leading knowledge translation and dissemination activities in partnership with Obesity Canada. Committee members with expertise in evidence syntheses and guideline development will be responsible for creating relevant content (e.g., summary of findings tables). In addition, caregivers, youth, clinicians, researchers and trainees with complementary experience and expertise will contribute to this work.

Five teams will be formed to complete literature reviews on the 5 topics listed below. These teams will include content experts in lead or colead roles and methodologic experts from MERST and AbSPORU.

\section{Selection of key questions}

To inform the guideline, literature reviews will be conducted to answer the following 5 questions, which the committee developed through numerous teleconferences and email discussions, following an iterative process (Table 1):

1. What are the values, preferences, perceptions, attitudes and beliefs of children and adolescents with obesity and their caregivers regarding the benefits and harms (in the context of important health-related outcomes) of obesity management strategies?

2. What tools, processes and procedures are recommended by expert groups for the clinical assessment of children and adolescents with obesity? What are the gaps in the existing guidelines?

3. Among children and adolescents with obesity, what is the effect of psychological and behavioural change interventions (see Appendix 1, available at www.cmajopen.ca/ content/10/1/E155/suppl/DC1, for definition) on health outcomes deemed important to stakeholders, including families, clinicians and researchers? 
4. Among children and adolescents with obesity, what is the effect of pharmacotherapeutic interventions on health outcomes deemed important to stakeholders, including families, clinicians and researchers?

5. Among children and adolescents with obesity, what is the effect of bariatric surgery interventions on health outcomes deemed important to stakeholders, including families, clinicians and researchers?
Of these 5 questions, 2 questions (values and preferences; clinical assessment) highlight contextual issues that are relevant to families and clinicians and 3 questions focus on primary interventions (psychological and behavioural; pharmacotherapeutic; and surgical) used in managing pediatric obesity.

We will conduct systematic reviews for questions 1, 3, 4 and 5 and an environmental scan and scoping review for question 2. The committee will operationalize the research questions for

Table 1 (part 1 of 2): Overview of literature reviews that will address research questions to inform the guideline

\begin{tabular}{|c|c|c|c|c|c|c|}
\hline Question & Review type & $\begin{array}{l}\text { Study } \\
\text { designs } \\
\text { included }\end{array}$ & Comparison & $\begin{array}{l}\text { Follow-up } \\
\text { duration } \\
\text { (after } \\
\text { baseline) }\end{array}$ & Potential outcomes* & $\begin{array}{c}\text { Potential subgroups } \\
\text { and sensitivity } \\
\text { analysis* }\end{array}$ \\
\hline $\begin{array}{l}\text { 1. What are values, } \\
\text { preferences, } \\
\text { perceptions, attitudes } \\
\text { and beliefs of children } \\
\text { and adolescents with } \\
\text { obesity and their } \\
\text { caregivers regarding } \\
\text { the benefits and } \\
\text { harms (in the context } \\
\text { of important } \\
\text { health-related } \\
\text { outcomes) of obesity } \\
\text { management } \\
\text { strategies? }\end{array}$ & $\begin{array}{l}\text { Systematic } \\
\text { review }\end{array}$ & Any & NA & NA & $\begin{array}{l}\text { Perceptions, } \\
\text { experiences, } \\
\text { attitudes, beliefs } \\
\text { and expectations }\end{array}$ & $\begin{array}{l}\text { - Children, } \\
\text { adolescents and } \\
\text { parents } \\
\text { - Sex, gender } \\
\text { - Ethnicity, culture, } \\
\text { SES } \\
\text { - Typical v. atypical } \\
\text { growth and } \\
\text { development } \\
\text { (physical or } \\
\text { cognitive delay or } \\
\text { disability) }\end{array}$ \\
\hline $\begin{array}{l}\text { 2. What tools, } \\
\text { processes and } \\
\text { procedures are } \\
\text { recommended by } \\
\text { expert groups for the } \\
\text { clinical assessment } \\
\text { of children and } \\
\text { adolescents with } \\
\text { obesity? What are the } \\
\text { gaps in the existing } \\
\text { guidelines? }\end{array}$ & $\begin{array}{l}\text { Scoping } \\
\text { review, with } \\
\text { stakeholder } \\
\text { consultation }\end{array}$ & Any & NA & NA & $\begin{array}{l}\text { - Edmonton Obesity } \\
\text { Staging System for } \\
\text { Pediatrics, }{ }^{23} \\
\text { including the } 4 \mathrm{Ms} \\
\text { (metabolic health, } \\
\text { mental health, } \\
\text { mechanical health } \\
\text { and social milieu) }\end{array}$ & $\begin{array}{l}\text { - Children, } \\
\text { adolescents, and } \\
\text { parents } \\
\text { - Sex, gender } \\
\text { - Ethnicity, culture, } \\
\text { SES } \\
\text { - Typical v. atypical } \\
\text { growth and } \\
\text { maturation (physical } \\
\text { or cognitive delay or } \\
\text { disability) } \\
\text { - Communication and } \\
\text { terminology } \\
\text { - Weight bias and } \\
\text { stigma } \\
\text { - Screening, } \\
\text { enrolment and } \\
\text { follow-up }\end{array}$ \\
\hline $\begin{array}{l}\text { 3. Among children } \\
\text { and adolescents with } \\
\text { obesity, what is the } \\
\text { effect of psychological } \\
\text { and behavioural } \\
\text { change interventions } \\
\text { (see Appendix } 1 \text { for } \\
\text { definition) on health } \\
\text { outcomes deemed } \\
\text { important to } \\
\text { stakeholders, } \\
\text { including families, } \\
\text { clinicians and } \\
\text { researchers? }\end{array}$ & $\begin{array}{l}\text { Systematic } \\
\text { review and } \\
\text { meta- } \\
\text { analysis }\end{array}$ & $\begin{array}{l}\text { Randomized } \\
\text { controlled } \\
\text { trials }\end{array}$ & $\begin{array}{l}\text { Any } \\
\text { nonactive } \\
\text { (e.g., wait-list } \\
\text { control) or } \\
\text { active (e.g., } \\
\text { standard } \\
\text { care) } \\
\text { alternative } \\
\text { management } \\
\text { strategies }\end{array}$ & $\begin{array}{l}\text { Immediate } \\
\text { post and } \\
\text { longest } \\
\text { follow-up } \\
\text { (closest to } \\
12 \text { mo) }\end{array}$ & $\begin{array}{l}\text { - Anthropometry } \\
\text { (e.g., body weight, } \\
\text { BMI, WC) } \\
\text { - Cardiometabolic } \\
\text { risk factors (e.g., } \\
\text { blood pressure, } \\
\text { insulin resistance, } \\
\text { HDL-C) } \\
\text { - Outcomes reported } \\
\text { by patients or } \\
\text { proxies (caregivers) } \\
\text { (e.g., anxiety, } \\
\text { depression, } \\
\text { health-related } \\
\text { quality of life) } \\
\text { - Adverse events }\end{array}$ & $\begin{array}{l}\text { - Age } \\
\text { - Weight status } \\
\text { - Sex, gender } \\
\text { - Risk of bias } \\
\text { - If we identify } \\
\text { studies that } \\
\text { reported data at } \\
\geq 16 \text { mo, we will } \\
\text { assess the 12-mo } \\
\text { estimate with and } \\
\text { without these data }\end{array}$ \\
\hline
\end{tabular}


Table 1 (part 2 of 2): Overview of literature reviews that will address research questions to inform the guideline

\begin{tabular}{|c|c|c|c|c|c|c|}
\hline Question & Review type & $\begin{array}{c}\text { Study } \\
\text { designs } \\
\text { included }\end{array}$ & Comparison & $\begin{array}{l}\text { Follow-up } \\
\text { duration } \\
\text { (after } \\
\text { baseline) }\end{array}$ & Potential outcomes* & $\begin{array}{c}\text { Potential subgroups } \\
\text { and sensitivity } \\
\text { analysis }{ }^{*}\end{array}$ \\
\hline $\begin{array}{l}\text { 4. Among children } \\
\text { and adolescents } \\
\text { with obesity, what } \\
\text { is the effect of } \\
\text { pharmacotherapeutic } \\
\text { interventions on } \\
\text { health outcomes } \\
\text { deemed important } \\
\text { to stakeholders, } \\
\text { including families, } \\
\text { clinicians and } \\
\text { researchers? }\end{array}$ & $\begin{array}{l}\text { Systematic } \\
\text { review and } \\
\text { meta- } \\
\text { analysis }\end{array}$ & $\begin{array}{l}\text { Randomized } \\
\text { controlled } \\
\text { trials }\end{array}$ & $\begin{array}{l}\text { Any } \\
\text { nonactive } \\
\text { (e.g., wait list } \\
\text { control) or } \\
\text { active (e.g., } \\
\text { standard } \\
\text { care) } \\
\text { alternative } \\
\text { management } \\
\text { strategies }\end{array}$ & $\begin{array}{l}\text { Immediate } \\
\text { post and } \\
\text { longest } \\
\text { follow-up } \\
\text { (closest to } \\
12 \text { mo) }\end{array}$ & $\begin{array}{l}\text { - Anthropometry } \\
\text { (e.g., body weight, } \\
\text { BMI, WC) } \\
\text { - Cardiometabolic } \\
\text { risk factors (e.g., } \\
\text { blood pressure, } \\
\text { insulin resistance, } \\
\text { HDL-C) } \\
\text { - Outcomes reported } \\
\text { by patients or } \\
\text { proxies (caregivers) } \\
\text { (e.g., anxiety, } \\
\text { depression, } \\
\text { health-related } \\
\text { quality of life) } \\
\text { - Adverse events }\end{array}$ & $\begin{array}{l}\text { - Age } \\
\text { - Weight status } \\
\text { - Sex, gender } \\
\text { - Risk of bias } \\
\text { - If we identify } \\
\text { studies that } \\
\text { reported data at } \\
\geq 16 \mathrm{mo} \text {, we will } \\
\text { assess the } 12-\text { mo } \\
\text { estimate with and } \\
\text { without these data }\end{array}$ \\
\hline $\begin{array}{l}\text { 5. Among children } \\
\text { and adolescents with } \\
\text { obesity, what is the } \\
\text { effect of bariatric } \\
\text { surgery interventions } \\
\text { on health outcomes } \\
\text { deemed important } \\
\text { to stakeholders, } \\
\text { including families, } \\
\text { clinicians and } \\
\text { researchers? }\end{array}$ & $\begin{array}{l}\text { Systematic } \\
\text { review and } \\
\text { meta- } \\
\text { analysis }\end{array}$ & $\begin{array}{l}\text { Randomized } \\
\text { controlled } \\
\text { trials, } \\
\text { prospective } \\
\text { or } \\
\text { retrospective } \\
\text { cohort } \\
\text { studies and } \\
\text { other } \\
\text { observational } \\
\text { studies }\end{array}$ & $\begin{array}{l}\text { Any } \\
\text { nonactive } \\
\text { (e.g., wait list } \\
\text { control) or } \\
\text { active (e.g., } \\
\text { standard } \\
\text { care) } \\
\text { alternative } \\
\text { management } \\
\text { strategies }\end{array}$ & $\begin{array}{l}\text { For the } \\
\text { weight } \\
\text { outcomes } \\
\text { up to } \\
12 \text { mo; } \\
18 \text {-mo and } \\
\text { longest } \\
\text { follow-up; } \\
\text { for other } \\
\text { outcomes } \\
\text { up to } \\
18 \text { mo }\end{array}$ & $\begin{array}{l}\text { - Anthropometry } \\
\text { (e.g., body weight, } \\
\text { BMI, WC) } \\
\text { - Cardiometabolic } \\
\text { risk factors (e.g., } \\
\text { blood pressure, } \\
\text { insulin resistance, } \\
\text { HDL-C) } \\
\text { - Outcomes reported } \\
\text { by patients or } \\
\text { proxies (caregivers) } \\
\text { (e.g., anxiety, } \\
\text { depression, } \\
\text { health-related } \\
\text { quality of life) } \\
\text { - Adverse events }\end{array}$ & $\begin{array}{l}\text { - Age } \\
\text { - Weight status } \\
\text { - Sex, gender } \\
\text { - Risk of bias } \\
\text { - If we identify } \\
\text { studies that } \\
\text { reported data at } \\
\geq 16 \mathrm{mo} \text {, we will } \\
\text { assess the 12-mo } \\
\text { estimate with and } \\
\text { without these data }\end{array}$ \\
\hline
\end{tabular}

the 3 intervention-related systematic reviews (questions 3, 4 and 5) regarding intervention characteristics, accepted comparators, ranking of outcomes by their relative importance to patients, and selection of subgroups on the basis of literaturederived, clinically relevant effect modifiers. For each of these intervention-related reviews, outcomes of interest will be determined using an online survey of stakeholders, including committee members and caregivers of children and adolescents enrolled in multidisciplinary obesity management clinics in several Canadian centres. These data will help to ensure the guideline is relevant and meaningful to its end users: families and clinicians. ${ }^{18}$ Survey participants will rate health outcomes of interest on the basis of the extent to which they believe the outcomes are important to decision-making for obesity management. Outcomes will be rated using the following rubric: 1-3 (not important for making a decision), 4-6 (important, but not critical for making a decision) and 7-9 (critical for making a decision). ${ }^{19}$ A detailed description of the scope and characteristics of eligible interventions and studies for each of the
3 intervention-related reviews will be determined iteratively through committee member discussions, considering available evidence on child, adolescent and family relevance, feasibility and acceptability.

\section{Summarizing the evidence}

The Knowledge Translation Platform within AbSPORU will lead and support activities related to the reviews of values and preferences and clinical assessment. The MERST team will lead the systematic reviews for the 3 intervention-related reviews.

For our systematic review on values and preferences, we will include systematic reviews, primary quantitative studies (such as surveys, health state value studies [e.g., standard gamble, time trade-off, visual analogue scale for a specific outcome], direct choice studies [e.g., choice when presented with decision aid, probabilistic trade-off techniques, conjoint analysis willingness to pay, randomized controlled trials on preferences]), qualitative studies (such as interviews, focus groups) and mixed-methods studies that include children and adolescents aged 0 to 18 years 
and their families. Studies included in this review will explore the values, preferences, perceptions, attitudes and beliefs of children and adolescents with obesity and their caregivers with an emphasis on the best estimates of benefits and harms (in the context of important health-related outcomes) of obesity management strategies, described below.

To inform the recommendations on the clinical assessment of children and adolescents with obesity, we will carry out several sequential steps. First, we will conduct an environmental scan of existing clinical practice guidelines and expert recommendations for the assessment of pediatric obesity. From these sources, a table detailing the processes, procedures and methods used to complete a clinical assessment will be created to identify unique and common elements, identify gaps in the evidence and inform the generation of the research question(s) that will guide a scoping review. Second, we will complete a scoping review that adheres to established scoping review methods as described by the Joanna Briggs Institute ${ }^{20}$ and the Preferred Reporting Items for Systematic Reviews and Meta-analyses (PRISMA) for Scoping Reviews (PRISMA-ScR) statement checklist. ${ }^{21}$ Third, we will evaluate the methodologic quality of the guidelines included in our scoping review using the Appraisal of Guidelines for Research and Evaluation AGREE II tool, which is widely recognized as the standard tool for evaluating practice guidelines. ${ }^{22}$ Finally, we will complete a stakeholder consultation (e.g., with caregivers and clinicians) to solicit feedback before finalizing and publishing the review. To provide a framework for the results of this review, information will be organized according to the $4 \mathrm{M}$ health risks (metabolic health, mental health, mechanical health and social milieu); this approach is based on the Edmonton Obesity Staging System for Pediatrics. ${ }^{23}$ Issues of communication, bias and stigma, screening and follow-up, as well as relevant subgroups, will be included.

For our 3 intervention-related reviews (psychological and behavioural; pharmacotherapeutic; and surgical), we will access data from randomized clinical trials (RCTs), including controlled clinical trials (CCTs), that include children and adolescents aged 0 to 18 years and have safety and effectiveness data for our interventions of interest, immediately after the intervention and at the longest point of follow-up (see Appendix 2, available at www.cmajopen.ca/content/10/1/E155/suppl/DC1, for details regarding analysis of time points and follow-up). For our review on surgical interventions, we will include observational studies in addition to RCTs and CCTs. On the basis of the committee members' experience, it is unlikely that many surgical studies will have randomly assigned participants to different study arms; in this emerging field of more intensive therapies for managing pediatric obesity, cohorts and case series have been more common, so a range of study designs will be included.

The MERST and AbSPORU evidence centre teams will review titles and abstracts in duplicate; articles marked for inclusion will proceed to full-text relevance testing. Full-text screening will be completed independently by 2 team members, with consensus required for inclusion or exclusion. Multiple publications for the same primary intervention in the same cohort will be merged. Standardized forms for data extraction and risk of bias will be developed, piloted and used by the team. Risk of bias assessments will be conducted using the Cochrane risk-of-bias tool for RCTs ${ }^{24}$ and the Risk of Bias in Non-randomized Studies - of Interventions (ROBINS-I) for observational studies with comparison groups ${ }^{25}$ and the CLARITY group's Tool to Assess Risk of Bias in Cohort Studies (http://help.magicapp.org/knowledgebase/ articles/327941-tool-to-assess-risk-of-bias-in-cohort-studies).

If interventions have multiple treatment arms, we will follow Cochrane guidance on avoiding unit of analysis errors and only the interventions that meet inclusion criteria will be extracted. ${ }^{24}$ Following GRADE guidance, we will use data from complete cases in the primary analysis and assess for risk of bias associated with missing outcome data. ${ }^{26}$ Conflicts will be resolved through discussion, and a biostatistician will independently verify all data extraction.

Piloted data extraction forms will be used to extract information on study characteristics (e.g., design, sample size), participant characteristics (e.g., age, ethnicity), intervention and comparison characteristics (e.g., duration, dose), outcomes (e.g., definition, measurement) and results (e.g., number of events). All extracted data will be independently verified by a second reviewer, with the outcome data verified by a third person.

We will follow methods presented in the Cochrane handbook, ensuring a rigorous approach to planning, conducting and reporting systematic reviews. ${ }^{24}$ Each systematic review and meta-analysis, when relevant, will follow PRISMA ${ }^{27}$ and report on the outcomes ranked as important or critically important on the basis of our stakeholder survey. For each review, we will develop a peer-reviewed, comprehensive search strategy in consultation with expert librarians (from McMaster University and the University of Alberta) to identify all relevant studies. PRISMA reporting guidance and registration of the protocols in International Prospective Register of Systematic Reviews (PROSPERO [https://www.crd.york. ac.uk/prospero/]) and the Open Science Framework (https:// osf.io/) will serve to increase the completeness, methodologic quality, transparency and reliability of our reviews. ${ }^{27}$

\section{Data analysis}

For our 3 intervention-related reviews, data will be summarized and random effects meta-analyses will be conducted when at least 2 studies that are sufficiently homogenous have defined and reported an outcome similarly. Depending on the number and types of studies and intervention arms, we may conduct a network meta-analysis. ${ }^{28,29}$ Estimates of effect, namely pooled relative risks and risk differences for dichotomous outcomes and weighted or standardized mean differences for continuous outcomes, will be generated with $95 \%$ confidence intervals. To optimize the interpretability of our results for stakeholders, in addition to presenting standardized mean differences for studies that report different outcomes that measure the construct, we will consider supplementary methods of presenting effect estimates, including the magnitude of effect, by presenting results in relation to the minimal important difference (e.g., $\geq 0.25$ reduction in body mass index $z$ score) when credible minimal important differences exist. ${ }^{30-33}$ 
For all meta-analyses with at least 10 studies, the potential for publication bias will be assessed using a funnel plot. ${ }^{34}$ Statistical heterogeneity in effect estimates across studies will be assessed by visual inspection of forest plots and assessment of the $I^{2}$ statistic, using thresholds recommended by Cochrane to determine degree of heterogeneity. ${ }^{35}$ Heterogeneity will be assessed by conducting subgroup analyses that may help to explain observed effects. Sensitivity analyses comparing studies rated as "lower" versus "higher" risk of bias will also be conducted. It is possible that effect estimates of interventions at higher risk of bias will be larger, exaggerating estimates of effect. ${ }^{36}$

Evidence on the clinical rationale for the potential differences in the effects of interventions across subgroups, including age, sex or gender, and weight status will be used to evaluate effect modification on the basis of subgroup credibility criteria. ${ }^{37,38}$ Supplementing our a priori subgroup analyses, we will also consider post hoc subgroup analyses on the basis of suggestions from our guideline panel (which will include some members of the steering committee) when voting on recommendations.

\section{Assessment of certainty of evidence}

For our 4 systematic reviews, we will use the GRADE approach to rate the certainty of the overall body of evidence for each outcome summarized. ${ }^{15-17}$ On an outcome-by-outcome basis, for a systematic review based on RCTs, the GRADE approach starts at high-quality evidence and considers the presence of the following factors as potential reasons to reduce certainty: risk of bias, inconsistency of results, indirectness of evidence, imprecision and publication bias. For a systematic review derived from observational studies, the evidence starts at low quality; unsystematic clinical observations start at very low quality. However, the certainty may increase when a large effect or a credible dose-response gradient exists or when all plausible confounding or other biases may be working against the observed effect. While the certainty of a body of evidence will often represent a continuum, GRADE ultimately categorizes the certainty into 1 of 4 categories (Box 1$){ }^{17}$

\section{Development of recommendations}

The committee will use evidence to decision (EtD) frameworks to move from evidence to recommendations. ${ }^{39}$ These frameworks will enable our committee to consider all relevant criteria and use evidence in a structured, transparent manner to inform decisions related to recommendations. The frameworks encourage examining and considering different perspectives (individual v. public health) when making recommendations. As such, the Canadian clinical practice guideline for managing pediatric obesity will take a perspective at the level of the individual patient (child, adolescent or caregiver).

The following criteria will be considered by committee members for each intervention for which a recommendation will be made: overall certainty of the evidence; desirable effects of the intervention (e.g., benefits); undesirable effects of the intervention (e.g., harms); the balance between benefits and harms; the values and preferences of children, adolescents and their caregivers; any incurred costs for the individual and their families; and intervention acceptability and feasibility. ${ }^{39}$
Committee members will follow a process of assessing the evidence for each criterion and making judgments (Appendix 3, available at www.cmajopen.ca/content/10/1/E155/ suppl/DC1). Guideline committee members will complete an electronic GRADE EtD framework survey to help them apply GRADE evidence summaries in a structured and transparent way to develop the final recommendations. ${ }^{39}$ Committee members will be asked to consider the evidence summaries for health outcomes; values and preferences; and the acceptability, feasibility and cost of a recommendation to change health behaviours, begin medication or undergo surgery. Consensus appraisal of evidence quality by reviewers will inform the level of evidence in these recommendations. During the final committee meeting, panel members will review the results of the GRADE EtD framework survey and consider the implications of those judgments for their recommendations.

This process will assist the committee to make final recommendations for or against the relevant interventions and to label the recommendations as strong or weak (also called conditional, discretionary or qualified). ${ }^{16}$ The strength of a recommendation will reflect the guideline committee's level of certainty that desirable consequences will outweigh undesirable consequences when the recommendation is adhered to across the range of patients for whom the recommendation is intended. A recommendation is less likely to be strong when desirable and undesirable consequences are closely balanced or when the certainty in estimates of effects is low. Similarly, when uncertainty exists in estimates of values and preferences, or when resource use is high, the strength of a recommendation is likely to be weak. When relevant, the guideline committee may formulate recommendations tailored for specific subgroups.

Consistent with the process used to develop the adult obesity guideline, ${ }^{13}$ our committee will vote on each recommendation. If fewer than $75 \%$ of committee members agree on a given recommendation, but the recommendation is on a topic that is deemed to be essential for inclusion, the recommendation will be included as a key message. In these circumstances, the lead authors of the respective reviews will modify the wording of the recommendation for review and approval by the steering committee, which will also approve all the final recommendations. Recommendations will be stated in a concise, clear and actionable manner, with justification provided for each recommendation. The implications of strong and weak recommendations for different end-users are presented in Table $2 .{ }^{15}$

\section{Internal and external review process}

To ensure that the guideline, recommendations and accompanying tools and resources for clinicians are useful and relevant, all materials will be reviewed, both internally and externally. Internally, our committee and the teams that are leading our knowledge syntheses will prepare and draft all documents. These materials will then be reviewed externally by a diverse group of stakeholders, including families, clinicians, researchers and administrators representing professional societies and governmental agencies. 


\begin{tabular}{|c|c|c|}
\hline Implications & Strong recommendation & Weak recommendation \\
\hline For patients & $\begin{array}{l}\text { Most people in this situation would want the } \\
\text { recommended course of action and only a small } \\
\text { proportion would not. }\end{array}$ & $\begin{array}{l}\text { Most people in this situation would want the suggested } \\
\text { course of action, but many would not. }\end{array}$ \\
\hline For clinicians & $\begin{array}{l}\text { Most people should receive the recommended course of } \\
\text { action. Adherence to this recommendation according to } \\
\text { the guideline could be used as a quality criterion or } \\
\text { performance indicator. Formal decision aids are not likely } \\
\text { to be needed to help people make decisions consistent } \\
\text { with their values and preferences. }\end{array}$ & $\begin{array}{l}\text { Clinicians should recognize that different choices will } \\
\text { be appropriate for different patients and that they must } \\
\text { help each patient arrive at a management decision } \\
\text { consistent with her or his values and preferences. } \\
\text { Decision aids may be useful to help people to make } \\
\text { decisions consistent with their values and preferences. } \\
\text { Clinicians should expect to spend more time with } \\
\text { patients when working toward a decision. }\end{array}$ \\
\hline For policy-makers & $\begin{array}{l}\text { The recommendation can be used to develop policy (e.g., } \\
\text { taxation of products high in sugar or salt). }\end{array}$ & $\begin{array}{l}\text { Policy-making will require substantial debates and } \\
\text { involvement of many stakeholders. Policies are also } \\
\text { more likely to vary between regions. Performance } \\
\text { indicators would have to focus on the fact that } \\
\text { adequate deliberation about the management options } \\
\text { has taken place. }\end{array}$ \\
\hline
\end{tabular}

When our work began to update the guideline, one of our first tasks was to contact professional associations and governmental representatives to inform them of our plan. These stakeholders have been kept up to date regarding our activities.

Additionally, we will establish a family advisory panel, including both adolescents and caregivers, to solicit their input on our recommendations and accompanying educational tools and resources. When it comes time for documents to be reviewed externally, we will draw on their experience and expertise to seek feedback before the documents are revised (if indicated), finalized and disseminated. Our family advisory panel will contribute value and preference information regarding the results of our 3 interventionrelated systematic reviews (psychological and behavioural; pharmacotherapeutic; and surgical), share perspectives and experiences relevant to our clinical assessment review, and provide guidance and input regarding our knowledge dissemination strategies.

We will strive to achieve consensus among stakeholders regarding our recommendations; however, for transparency, we will document any differences of opinion in perspective (e.g., acceptability, feasibility) within our GRADE EtD summary tables and our summary guideline document. If consensus cannot be achieved on a recommendation, it will be considered (and revised accordingly) for inclusion as a key message. A final step will include working with editorial and design professionals to harmonize the style and formatting of all relevant materials.

\section{Management of competing interests}

On an annual basis, each member of the guideline committee will complete a written declaration of potential competing interests using the International Committee of Medical Journal Editors' disclosure form (http://www.icmje.org/disclosure -of-interest/). As circumstances and opportunities can change over time, at the beginning of each monthly teleconference, committee members will be prompted to report any changes to their competing interests statement. The committee will discuss and decide whether any disclosed competing interests are acceptable and, when applicable, how they will be managed with respect to each review and recommendation. In lieu of holding a formal vote, the committee will discuss issues and resolve them collaboratively to achieve consensus.

If the chair has a competing interest that cannot be resolved through discussion, and to determine which committee members hold voting and nonvoting roles in establishing intervention-specific recommendations, our oversight subcommittee will convene, which will include 1 clinicianscientist, 1 methods expert, 1 caregiver representative and 1 stakeholder from Obesity Canada, to discuss and determine how the competing interest(s) will be addressed. Obesity Canada has developed and will manage for this project a competing interest policy and procedures for mitigating bias.

Individuals with relevant disclosures will not be excluded from conducting the critical appraisals or voting on recommendations. However, individuals with direct competing interests will be asked to abstain from voting in the areas in which they have the conflict. Our plan to manage committee members' competing interests adheres to principles endorsed by the Guidelines International Network. ${ }^{40}$

Any discussion regarding off-label use of drugs will include the caveat that the use is off-label. The views of the funding bodies (Obesity Canada FOCUS Fund, Alberta Health Services) will have no influence over the content of the guideline. All committee members will be volunteers and will not be remunerated for their services.

\section{Ethics approval}

Our survey of stakeholders was approved by human research ethics boards at the University of Alberta (Edmonton), The Hospital for Sick Children (Toronto), and the Children's Hospital of Eastern Ontario (Ottawa). 


\section{Interpretation}

The process of developing this guideline will bring together families living with obesity as well as multidisciplinary clinicians from across Canada to make informed, value-sensitive, evidence-based recommendations for managing pediatric obesity based on systematic reviews of the literature and internationally recognized guideline standards. We will produce health-related recommendations that are based on values and preferences expressed by families and informed by systematic reviews of obesity management strategies related to our target health outcomes.

Knowledge translation products and tools, with a focus on integration in clinical practice and dissemination of evidence and recommendations, will be fundamental to the success of the guideline recommendations. By including stakeholders (families, clinicians) in all activities and communicating and meeting regularly with committee members and external stakeholders, we will aim to ensure the guideline is responsive and relevant. Obesity Canada, which is sponsoring the guideline through in-kind contributions, will strive to integrate knowledge translation into the guideline development process by collaborating with knowledge translation experts as well as decision-makers, including patients, families and community members. Mechanisms to translate science to the public include a plain-language, bilingual website, blog series, infographics and whiteboard videos, public workshops, webinars, social media activities and a growing online support community for people living with obesity.

Through their public engagement and expertise in knowledge translation, Obesity Canada will support this guideline and share it with its network of health professionals and people living with obesity, integrate the guideline into its education programs designed to develop skills among clinicians and families affected by obesity, and disseminate the key findings to decision-makers across the country through its ongoing advocacy activities.

To optimize uptake, the guideline and companion documents for families and clinicians will be prepared originally in English, then translated into French. Obesity Canada will consult with federal, provincial and territorial decision-makers to identify barriers and facilitators for implementation.

\section{Limitations}

Our guideline will not be without limitations. First, we will address only obesity assessment and management. Given the large evidence base on potential preventive measures for obesity, and the resources required to coordinate a timely series of systematic reviews and linked recommendations for both prevention and management, our guideline will focus exclusively on the management of pediatric obesity. Second, as per the National Academy of Medicine standards, ${ }^{14}$ guidelines should be regularly updated. Although we have not yet secured funding to update our recommendations regularly, we intend to continue this work. We have communicated with the World Health Organization and the American Academy of Pediatrics to explore the possibility of developing a jointly funded guideline in the future, which may improve efficiency and avoid duplication of effort. Third, we considered multiple outcomes and subgroups of interest for each of our 3 systematic reviews on interventions. On the basis of GRADE guidance ${ }^{17}$ and our survey of families and clinicians managing obesity, we will limit our outcomes to the ones deemed to be most important to our stakeholders. Our subgroups will be selected on the basis of the most robust evidence for effect modification, while leaving room for post hoc subgroup analyses based on guideline panel suggestions when voting on recommendations.

\section{Conclusion}

An updated, national guideline for assessing and managing pediatric obesity in Canada is needed because of the publication of relevant new evidence and the evolution of methods and standards for the development of trustworthy guidelines over the past decade. We present methods for the development of a guideline that adhere to the standards set by leading medical and methodologic groups and describe the guideline oversight and leadership structure, the approach to strict management of competing interests, the engagement of a diverse panel of stakeholders and the methods that will be used to synthesize existing evidence and to move from evidence to recommendations. Once published, the guideline will support children and adolescents, their caregivers and clinicians in Canada in making informed, value-sensitive and evidence-based clinical decisions related to the management of pediatric obesity.

\section{References}

1. NCD Risk Factor Collaboration (NCD-RisC). Worldwide trends in children's and adolescents' body mass index, underweight, overweight and obesity, in comparison with adults, from 1975 to 2016: a pooled analysis of 2,416 population-based measurement studies with 128.9 million participants. Lancet 2017;390:2627-42.

2. Ball GDC, Savu A, Kaul P. Changes in the prevalence of overweight, obesity, and severe obesity between 2010 and 2017 in preschoolers: a populationbased study. Pediatr Obes 2019;14:e12561.

3. Carsley S, Tu K, Parkin PC, et al. Overweight and obesity in preschool aged children and risk of mental health service utilization. Int 7 Obes (Lond) 2019;43:1325-33.

4. Rao DP, Kropac E, Do MT, et al. Childhood overweight and obesity trends in Canada. Health Promot Chronic Dis Prev Can 2016;36:194-8.

5. Hadjiyannakis S, Ibrahim Q, Li J, et al. Obesity class versus the Edmonton Obesity Staging System for Pediatrics to define health risk in childhood obesity: results from the CANPWR cross-sectional study. Lancet Child Adolesc Health 2019;3:398-407.

6. Freedman DS, Lawman HG, Galuska DA, et al. Tracking and variability in childhood levels of BMI: the Bogalusa Heart Study. Obesity (Silver Spring) 2018;26:1197-202.

7. Cunningham SA, Datar A, Narayan KMV, et al. Entrenched obesity in childhood: findings from a national cohort study. Ann Epidemiol 2017;27:435-41.

8. Reilly JJ, Kelly J. Long-term impact of overweight and obesity in childhood and adolescence on morbidity and premature mortality in adulthood: systematic review. Int 7 Obes (Lond) 2011;35:891-8.

9. Lau DCW, Douketis JD, Morrison KM, et al.; Obesity Canada Clinical Practice Guidelines Expert Panel. 2006 Canadian clinical practice guidelines on the management and prevention of obesity in adults and children [summary]. CMAf 2007;176:S1-13.

10. Canadian Task Force on Preventive Health Care. Recommendations for growth monitoring, and prevention and management of overweight and obesity in children and youth in primary care. CMAJ 2015;187:411-21.

11. Valerio G, Maffeis C, Saggese G, et al. Diagnosis, treatment and prevention of pediatric obesity: consensus position statement of the Italian Society for Pediatric Endocrinology and Diabetology and the Italian Society of Pediatrics. Ital 7 Pediatr 2018;44:88. 
12. Styne DM, Arslanian SA, Connor EL, et al. Pediatric obesity: assessment, treatment, and prevention - an Endocrine Society Clinical Practice guideline. 7 Clin Endocrinol Metab 2017;102:709-57.

13. Wharton S, Lau DCW, Vallis M, et al. Obesity in adults: a clinical practice guideline. CMA7 2020;192:E875-91.

14. Institute of Medicine (US) Committee on Standards for Developing Trustworthy Clinical Practice Guidelines. Clinical guidelines we can trust. Graham R, Mancher M, Miller Wolman D, et al., editors. Washington (DC): National Academies Press; 2011.

15. Andrews J, Guyatt G, Oxman AD, et al. GRADE guidelines: 14. Going from evidence to recommendations: the significance and presentation of recommendations. 7 Clin Epidemiol 2013;66:719-25.

16. Andrews JC, Schünemann HJ, Oxman AD, et al. GRADE guidelines: 15 . Going from evidence to recommendation-determinants of a recommendation's direction and strength. 7 Clin Epidemiol 2013;66:726-35.

17. Guyatt GH, Oxman AD, Vist GE, et al. GRADE: an emerging consensus on rating quality of evidence and strength of recommendations. BMF 2008; 336:924-6.

18. G-I-N Public Toolkit: patient and public involvement in guidelines. Berlin (Germany): Guidelines International Network (G-I-N); 2015. Available: https://g-i-n.net/ new-digital-gin-public-toolkit-patient-and-public-involvement-in-guidelines/ (accessed 2021 Nov. 25).

19. Schünemann H, Brozek J, Guyatt G, et al. GRADE handbook for grading quality of evidence and strength of recommendations. Version 3.2. GRADE Working Group; updated 2013. Available: https://gdt.gradepro.org/app/handbook/ handbook.html (accessed 2021 Nov. 25).

20. Peters MDJ, Godfrey C, McInerney P, et al. Chapter 11: Scoping reviews (2020 version). In: Aromataris E, Munn Z, editors. FBI manual for evidence synthesis. Adelaide (AU): Joanna Briggs Institute; 2020. Available: https://jbi-global -wiki.refined.site/space/MANUAL/3283910770/Chapter+11\%3A+Scoping+ reviews (accessed 2021 Nov. 25).

21. Tricco AC, Lillie E, Zarin W, et al. PRISMA extension for scoping reviews (PRISMA-ScR): checklist and explanation. Ann Intern Med 2018;169:467-73.

22. Brouwers MC, Kerkvliet K, Spithoff K; AGREE Next Steps Consortium. The AGREE Reporting Checklist: a tool to improve reporting of clinical practice guidelines. $B M \mathcal{F}$ 2016;352:11152.

23. Hadjiyannakis S, Buchholz A, Chanoine J-P, et al. The Edmonton Obesity Staging System for Pediatrics: a proposed clinical staging system for paediatric obesity. Paediatr Child Health 2016;21:21-6.

24. Higgins JPT, Thomas J, Chandler J, et al., editors. Cochrane handbook for systematic teviews of interventions. Version 6.0. London (UK): Cochrane; 2019. Available: https://training.cochrane.org/handbook/archive/v6 (accessed 2021 Nov. 25).

25. Sterne JA, Hernan MA, Reeves BC, et al. ROBINS-I: a tool for assessing risk of bias in non-randomised studies of interventions. BM7 2016;355:i4919.

26. Zhang Y, Coello PA, Guyatt GH, et al. GRADE guidelines: 20. Assessing the certainty of evidence in the importance of outcomes or values and preferences-inconsistency, imprecision, and other domains. 7 Clin Epidemiol 2019; 111:83-93.

27. Moher D, Liberati A, Tetzlaff J, et al. PRISMA Group. Preferred reporting items for systematic reviews and meta-analyses: the PRISMA statement. $\mathcal{F}$ Clin Epidemiol 2009;62:1006-12.

28. Hutton B, Salanti G, Caldwell DM, et al. The PRISMA extension statement for reporting of systematic reviews incorporating network meta-analyses of health care interventions: checklist and explanations. Ann Intern Med 2015; 162:777-84.

29. Li T, Puhan MA, Vedula SS, et al.; Ad Hoc Network Meta-analysis Methods Meeting Working Group. Network meta-analysis-highly attractive but more methodological research is needed. BMC Med 2011;9:79.

30. Ge L, Sadeghirad B, Ball GDC, et al. Comparison of dietary macronutrient patterns of 14 popular named dietary programmes for weight and cardiovascular risk factor reduction in adults: systematic review and network metaanalysis of randomised trials. BM7 2020;369:m696.

31. Ebrahim S, Vercammen K, Sivanand A, et al. Minimally important differences in patient or proxy-reported outcome studies relevant to children: a systematic review. Pediatrics 2017;139:e20160833.

32. Johnston BC, Alonso-Coello P, Friedrich JO, et al. Do clinicians understand the size of treatment effects? A randomized survey across 8 countries. CMAJ 2016;188:25-32.

33. Thorlund K, Walter SD, Johnston BC, et al. Pooling health-related quality of life outcomes in meta-analysis: a tutorial and review of methods for enhancing interpretability. Res Synth Methods 2011;2:188-203.

34. Sterne JAC, Sutton AJ, Ioannidis JPA, et al. Recommendations for examining and interpreting funnel plot asymmetry in meta-analyses of randomised controlled trials. BM7 2011;343:d4002.

35. Higgins JP, Thompson SG, Deeks JJ, et al. Measuring inconsistency in metaanalyses. BMF 2003;327:557-60.

36. Savovic J, Turner RM, Mawdsley D, et al. Association between risk-of-bias assessments and results of randomized trials in Cochrane reviews: the ROBES Meta-Epidemiologic Study. Am 7 Epidemiol 2018;187:1113-22.
37. Sun X, Ioannidis JPA, Agoritsas T, et al. How to use a subgroup analysis: users' guide to the medical literature. FAMA 2014;311:405-11.

38. Sun X, Briel M, Busse JW, et al. Subgroup Analysis of Trials Is Rarely Easy (SATIRE): a study protocol for a systematic review to characterize the analysis, reporting, and claim of subgroup effects in randomized trials. Trials 2009;10:101.

39. Alonso-Coello P, Oxman AD, Moberg J, et al.; el GRADE Working Group. GRADE Evidence to Decision (EtD) frameworks: a systematic and transparent approach to making well informed healthcare choices. 2: Clinical practice guidelines [article in Spanish]. Gac Sanit 2018;32:167.e1-e10.

40. Schünemann HJ, Al-Ansary LA, Forland F, et al.; Board of Trustees of the Guidelines International Network. Guidelines International Network: principles for disclosure of interests and management of conflicts in guidelines. Ann Intern Med 2015;163:548-53.

Competing interests: Bradley Johnston is a GRADE working group member and has received a grant from Texas A\&M AgriLife Research to fund investigator-initiated research related to saturated and polyunsaturated fats. The grant was from Texas A\&M AgriLife institutional funds from interest and investment earnings, not a sponsoring organization, industry or company. Diana Sherifali has worked as a consultant for Obesity Canada and received travel support to attend a meeting with the World Heart Federation. Catherine Birken has received research funding from the Canadian Institutes of Health Research; the Heart and Stroke Foundation of Canada; Physician Services, Inc.; the Leong Centre, University of Toronto and Hospital for Sick Children; the Centre for Addiction and Mental Health; and a Walmart community grant through the SickKids Foundation. Jill Hamilton has received research funding from Mead Johnson, Levo Therapeutics and the Heart and Stroke Foundation of Canada. She received an honorarium from Pfizer Canada for a sponsored lecture on Prader-Willi Syndrome and participated as a member of the Novo Nordisk Canada national advisory board on pediatric obesity. Dawn Hatanaka is employed by Obesity Canada, which contributed in-kind resources to support this project. Mélanie Henderson holds a Fonds de la recherche du Québec - Santé (FRQS) Junior 2 Clinical Research Scholar Award. She is a member of Canada's Children's Hospitals Foundations (CCHF) and the Sun Life Child and Youth Type 2 Diabetes Prevention initiative. She also sits on the Comité scientifique sur la prévention de l'obésité of the Institut national de santé publique du Québec and the Groupe de consultation de la prise en charge de l'obésité chez l'enfant et l'adolescent and Comité consultatif québécois en médecine bariatrique of the Quebec Ministry of Health (no financial support). She holds research funding from the Canadian Institutes of Health Research, the Quebec Cardiometabolic Health, Diabetes and Obesity Research Network and the Quebec Network on Nursing Intervention Research, Diabète Québec, as well as through the CCHF-Sun Life initiative. Sarah Moore has received research funding from the Michael Smith Foundation for Health Research, the Natural Sciences and Engineering Research Council of Canada and the Kids Brain Health Network. She currently holds startup research funding from the Faculty of Health at Dalhousie University. Katherine Morrison has received consulting fees from Novo Nordisk Canada and research funding from the Canadian Institutes of Health Research. Ximena Ramos Salas has received consulting fees from Obesity Canada, the European Association for the Study of Obesity and the World Health Organization Regional Office for Europe. She has also received research funding from the Canadian Institutes of Health Research. Ian Zenlea has received an honorarium from Novo Nordisk Canada for participating as a member of the pediatric obesity national advisory board and for participating in a meeting to discuss diagnostic approaches to short stature in Canada, as well as an honorarium from Abbott Diabetes Care for participating as a member of a national advisory board. He has also received speaker fees from the Ontario Association of Social Workers. He has received research funding from the Canadian Institutes of Health Research, the University of Toronto, the Social Sciences and Humanities Research Council of Canada, the Medical Psychiatry Alliance and the Regional Municipality of Peel. He is co-chair of the Ontario Paediatric Bariatric Network. Geoff Ball has received support from Novo Nordisk Canada for participating as a member of the pediatric obesity national advisory board, in-kind resources from Obesity Canada, research and administrative support from Alberta Health Services (AHS; AHS Chair in Obesity Research) and research funding from the Canadian Institutes of Health Research, Alberta Innovates, the AHS Strategic Clinical Network (Maternal, Newborn, Child \& Youth), the Public Health Agency of Canada and the Women and Children's Health Research Institute of the University of Alberta. No other competing interests were declared. 
Affiliations: Departments of Nutrition and of Epidemiology and Biostatistics (Johnston), Texas A\&M University, College Station, Tex.; Department of Community Health and Epidemiology (Johnston, Merdad), Dalhousie University, Halifax, NS; School of Nursing (Sherifali), McMaster University, Hamilton, Ont.; Pennington Biomedical Research Center (Kebbe), Louisiana State University, Baton Rouge, La.; Department of Paediatrics (Birken, Hamilton), University of Toronto, Toronto, Ont.; Children's Hospital of Eastern Ontario Research Institute (Buchholz), Ottawa, Ont.; Department of Psychology (Buchholz), Carleton University, Ottawa, Ont.; Evidence-based Social Science Research Centre (Ge), School of Public Health, Lanzhou University, Lanzhou, China; School of Public Health (Gehring), University of Alberta, Edmonton, Alta.; Department of Pediatrics (Hadjiyannakis), Faculty of Medicine, University of Ottawa, Ottawa, Ont.; Obesity Canada (Hatanaka, Ramos Salas), Edmonton, Alta.; Department of Pediatrics (Henderson), Université de Montréal, Montréal, Que.; Centre de recherche du Centre hospitalier universitaire Sainte-Justine (Henderson), Montréal, Que.; Department of Social and Preventive Medicine (Henderson), School of Public Health, Université de Montréal, Montréal, Que.; patient and family partner (Lebel), Edmonton, Alta.; School of Health \& Human Performance (Moore), Faculty of Health, Dalhousie University, Halifax, NS; Department of Pediatrics (Morrison), McMaster University, Hamilton, Ont.; Department of Pediatrics (Sebastianski, Ball), University of Alberta, Edmonton, Alta.; Institute for Better Health (Zenlea), Trillium Health Partners, Mississauga, Ont.

Contributors: Bradley Johnston and Roah Merdad were joint primary authors. Bradley Johnston, Roah Merdad, Maryam Kebbe and Geoff Ball cowrote the first draft of the manuscript. Diana Sherifali, Catherine Birken, Annick Buchholz, Long Ge, Nicole Gehring, Stasia Hadjiyannakis, Jill Hamilton, Dawn Hatanaka, Melanie Henderson, Tracy Lebel, Sarah Moore, Katherine Morrison, Ximena Ramos Salas, Meghan Sebastianski and Ian Zenlea revised the manuscript for important intellectual content. All authors helped to conceive and design the work, approved the final version to be published and agree to be accountable for all aspects of the work in ensuring that questions related to the accuracy or integrity of any part of the work are appropriately investigated and resolved.

Funding: No funding was received for the preparation of this protocol.

Content licence: This is an Open Access article distributed in accordance with the terms of the Creative Commons Attribution (CC BY-NC-ND 4.0) licence, which permits use, distribution and reproduction in any medium, provided that the original publication is properly cited, the use is noncommercial (i.e., research or educational use), and no modifications or adaptations are made. See: https://creativecommons.org/licenses/ by-nc-nd/4.0/

Data sharing: Data will be shared upon reasonable request to the corresponding author after data collection has been completed.

Acknowledgements: The authors wish to thank Christina Giese (University of Alberta) and Nicole Pearce (Obesity Canada) for their clerical and logistical support for this project.

Supplemental information: For reviewer comments and the original submission of this manuscript, please see www.cmajopen.ca/content /10/1/E155/suppl/DC1. 